\title{
The Ecological Dimension of Natural Selection
}

\author{
Abstract: In this paper I argue that we should pay extra attention to the ecological \\ dimension of natural selection. By this I mean that we should view natural \\ selection primarily as acting on the outcomes of the interactions organisms have \\ with their environment which influences their relative reproductive output. A \\ consequence of this view is that natural selection is not (directly) sensitive to \\ what system of inheritance which ensures reoccurrences of organism- \\ environment interactions over generations. I end by showing the consequences \\ of this view when looking at how processes like niche construction and the \\ Baldwin effect relate to natural selection.
}


1. Introduction. The principle of natural selection is the theoretical cornerstone of evolutionary theory. In the philosophy of biology, we can delineate four different, but related, main discussions of this principle; first, on what the sufficient conditions are for its occurrence (e.g., Lewontin 1970; Godfrey-Smith 2009). Second, on the appropriate means of quantifying the influence of natural selection on the distribution of variants in populations over time (e.g., Millstein 2009; Otsuka 2016). Third, on whether selection can be counted as a cause or is more appropriately interpreted as a statistical summary of multiple underlying causes and not a cause of evolution in itself (e.g., Matthen and Ariew 2002; Ramsey 2013ab; Walsh 2010). Fourth, on whether selection can act on multiple levels and what the relevant units of selection are, and if any of these are privileged (e.g., Williams 1966; Dawkins 1976; Okasha 2006).

Another debate, which is related to all of the aforementioned debates, centers around the metaphysics of evolution. In this debate we can identify two main camps; a molecular, or "gene-centered" metaphysics (e.g., Dawkins 1976, 1982) and an ecological, or "organism-centered" metaphysics (e.g., West-Eberhard 2003; Walsh 2015). Standard textbook evolutionary biology usually has a "molecular" metaphysics, in that the fundamental units of evolution are genes. On an "ecological" metaphysics of evolution, the fundamental unit of evolution are organisms.

Walsh (2015), amongst others (see references below), has recently argued that the Modern Synthesis misrepresents the metaphysics of evolution by viewing it primarily as a molecular phenomenon, instead of an ecological one. This is largely due to what Walsh 
calls "the marginalisation of the organism that have taken hold under the Modern Synthesis" (Walsh 2015, x). This has been a complaint of many biologists and philosophers over the last decades (e.g., Lewontin 1983, Piaget 1978; Odling-Smee et al. 2003; Oyama 2000; West-Eberhard 2003) and is a central complaint of the proponents of an extended evolutionary synthesis (Pigliucci and Müller 2010). Theoretical and empirical work taking a more ecological or organism-centered approach to understanding evolution and development has also recently gained some traction under the headings of eco-devo (ecological developmental biology) and eco-evo-devo (ecological evolutionary developmental biology). For example, West-Eberhard (2003), Sultan (2015) and Gilbert and Epel (2015) have made a great effort to establish how both evolutionary and developmental trajectories are significantly influenced by, and sometimes crucially dependent on, particular organism-environment interactions.

This paper is a philosophical contribution to what an "organism-centered", or "ecological", metaphysics of evolution might do to our understanding of natural selection. I begin from the view that natural selection is primarily an ecological process. By this I mean that natural selection is a process where organism-environment interactions are what is preferentially selected. Further, natural selection acts on the outcomes of these interactions. This is not a novel view and has been suggested before (Lehrman 1970; Brandon 1990; Rosenberg 1983). However, I will take this a step further and argue that this also means that natural selection is not directly sensitive to which system of inheritance ensures the reoccurrence of such interactions, be it genetic, epigenetic, behavioral, cultural, or symbolic (Jablonka and Lamb 2014). Natural selection acts on the 
outcomes of organism-environment interactions and the frequency and likelihood of their reoccurrence in subsequent generations.

However, this does not mean that I equate the importance of each system of inheritance. A genetic system of inheritance is an important prior condition for there to be other systems of inheritance in most, if not all, organisms. Further, most of morphological and physiological evolution seem to be primarily under genetic control. The point is rather that this happens "unbeknownst" to natural selection. To use some helpful terminology from Sober (1984), we can say that there is selection for the ecological interactions that yields highest relative fitness in a population, while there is selection of the relevant genes that contribute to those interactions because of the high-fidelity-inheritance properties of the genetic system of inheritance in reliably producing offspring which have similar interactions.

2. Selection on Passive Objects by Environmental Filtration. Let us begin by looking in more detail at the "standard" molecular metaphysics of the Modern Synthesis. In most textbooks on evolutionary biology, one is likely to find a definition of evolution as the changes to allele (or gene) frequencies in a population over time (e.g., Futuyma and Kickpatrick 2017). Furthermore, the conditions for evolution by natural selection to occur (e.g., Lewontin 1970); inheritance, variation, and differences in fitness, is often interpreted in a genetic manner. That is, any variation in fitness, which is due to differences in the performance of varying phenotypes in relation to the local (and shared) selective environment, is only acted on by natural selection insofar as the genetic underpinning of 
that variation steadily expresses the relevant phenotype over generations. Since the genetic system of inheritance is privileged, in the sense that without it there would be (in most cases) no organism to be selected for in the first place, it makes perfect sense to define evolution as changes in the frequencies of genes in a population. And from this it easy to conceive of natural selection as being an agent which sorts different genetic variants based on their performance relative to their immediate environment. This rendition of natural selection construes it as an environmental process. The metaphor of a sieve or filtration is often invoked to describe this process (e.g., Sober 1984). Coupled with the view that the only phenotypic variation that matters for biological evolution is that which is the result of genetic variation, such metaphors engender a certain passivity on behalf of the organism. It essentially relegates the action of selection to be realized by certain (stable or changing) environmental configurations. Natural selection acts on those organisms that carry the appropriate genetic material to produce a phenotype that performs best (i.e., highest realized relative fitness) in relation to the relevant environmental configurations. Such a view of evolution by natural selection has been called asymmetrically externalist (GodfreySmith 1996). It is asymmetric in the sense that the configurations of the environment are (presumed to be) explainable solely with reference to factors internal to the environmental system itself. While, on the other hand, the organisms which occupy these environments are explained (in terms of the phylogenetic history leading up to their capacity for occupying the environment) by reference to a combination of changes to the biological system (i.e., changes in the gene frequencies of the lineage(s) leading up to the relevant population) and the environmental configuration which the lineage(s) have experienced 
over generations. It is externalist in the sense that the environmental configurations are what "trigger" the selection of the phenotype, while the changes to the gene frequencies in the population is a "structuring" cause of the selection event. ${ }^{1}$ The role of the organism in such explanations is that of a vehicle (Dawkins 1978), one that carries certain passengers (genes) to certain destinations (selection events). However, organisms are arguably not just an ensemble of genes, and their activity or behavior might influence their reproductive success and consequently the evolution of their lineage. How does an externalist and molecular (i.e., gene-centered) view of evolution deal with behavior?

Standardly, in behavioral ecology (e.g., Krebs and Davies 1993) and the evolutionary explanations provided by behavioral genetics (e.g., Anholt and Mackay 2010), organismic activity and behavior is treated as any other phenotypic trait. It is based on certain assumptions regarding the dispositional properties of genes in relation to behaviors and certain optimality measures (Krebs and Davies 1993). Generally speaking, organisms exhibiting behaviors that increase their fitness are selected for, and the disposition to exhibit the beneficial behavior in subsequent generations is assumed to be under genetic control — and can consequently be treated like any other phenotypic trait. The validity of these assumptions is not under question here. The point here is a conceptual one. It is about how we conceive of the relation between natural selection and the organisms exhibiting the relevant behavior. Let us do a thought experiment. Take an

\footnotetext{
${ }^{1}$ For the distinction between "structuring" and "triggering" causes, see Dretske (1988). For an example of its relevance for evolutionary theory, see Ramsey (2016).
} 
imaginary species like the tarbutniks from Avital and Jablonka (2000). The individuals of this species have completely identical and non-changing genetic make-up. In other words, it is a species without genetic variation among the individuals. However, let us assume that they can differ in their behavior, i.e. that there is still phenotypic variation. Let us then imagine that some individuals forage fruits to supplement their diet, while others obtain their nutrients from only eating grass. This then leads to the fruit-foraging individuals having a more energy-rich diet, which increases their reproductive output. Let us further imagine that the fruit foraging techniques are passed on vertically through parental guidance (i.e., learning) and that the transmission of this behavior from parent to offspring enjoys a high level of fidelity. If we view natural selection as a process that sorts genetic variation, then there is no response to selection in this scenario. However, this seems wrong. Surely, natural selection still acts on the individuals that forage fruit to supplement their diet if this increases their reproductive output. Thus, there is a response to selection in the population - the number of fruit-foraging individuals increases and fruit-foraging behavior spreads throughout the population.

While in this thought experiment natural selection does not lead to biological evolution (in the sense that the gene frequencies in the population remain unchanged), natural selection has still occurred. And while it might be true that for natural selection to bring about adaptive biological evolution there must selection amongst different genetic variants in a population, there is still natural selection amongst the phenotypes of our imagined population. The strength and direction of the selection for the fruit foraging behavior is dependent on the fidelity and transience of the behavioral inheritance system. 
Even though there are no organisms like the tarbutniks in the real world and we do not know exactly to what extent difference in behavior and capacity for learning is linked to and/or governed by genetic variation in a population, the point about the natural selection being an ecological process still stands. Natural selection is not directly sensitive to what causes the phenotypic variation available for selection to act on, just the outcome of different interactions between phenotypes and their environments. This is an important consideration for both biologists and philosophers taking a more organism-centered approach. These argue that organisms are not merely passive objects of selection, but active subjects — or agents — in their own evolution (e.g., Lewontin 1983; Odling-Smee 2003; Bateson 2004). Let us now turn to these organism-centered views, and in particular two processes where the activities of organisms play an important part in shaping evolutionary dynamics - the Baldwin effect and niche construction.

3. Organisms as Agents in Evolutionary Theory. Over the course of the last decades there has been an increasing tension in evolutionary biology, culminating in an overarching debate surrounding whether an extended evolutionary synthesis is needed (Müller and Pigliucci 2010, Laland et al. 2014; Wray et al. 2014). A central part of this debate concerns the role that behavior, and organismic activity more generally, has on evolutionary dynamics. The question of how the activities and behaviors of organisms can alter the action of natural selection has a long history. It could, arguably, be said to date all the way back to Lamarck (Avital and Jablonka 2000). Alternatively, we can trace it back to the introduction of organic selection (also called the Baldwin effect) in the late $19^{\text {th }}$ century 
(Baldwin 1896a, 1896b; Morgan 1896; Osborn 1896). Organic selection refers to an evolutionary process that can turn acquired characters into congenital ones. More precisely, it refers to a three-step process; first, organisms can through their interactions with the environment systematically produce behavioral, morphological, or physiological modifications that are not hereditary, but increase the fitness of the organism that acquires them. Second, there is genetic variation in the population producing hereditary characters similar to characters that are acquired by the organisms through their environmental interactions. Third, this genetic variation is acted on by natural selection and subsequently spread in the population over the course of generations. The character was initially individually acquired, but is in time turned into a hereditary character (Simpson 1953). This process has recently garnered more attention in evolutionary biology. In the works of the late Patrick Bateson (2004, 2017a, 2017b; Bateson and Gluckman 2011) this process is revisited in light of what we have learned about social learning, transmission and nongenetic systems of inheritance over the last decades. Bateson refers to the Baldwin effect as the adaptability driver (Bateson 2017a). By this he means that, more often than what we initially may have thought, behavioral plasticity (behavior which is the result of stimuli or interactions with the environment, and not determined by genetic factors) is actually crucial in initiating adaptive responses to environmental challenges. ${ }^{2}$

\footnotetext{
${ }^{2}$ A more general rendition of this view, where not only behavioral but also morphological and physiological acquired characters are what initiates evolutionary change, is referred to as 'plasticity-first evolution' (e.g. Levis and Pfennig 2016).
} 
Another example of organismic activity altering evolutionary dynamics can be seen in niche construction theory (Odling-Smee 2003). Niche construction refers to cases where organisms modify selection pressures by actively altering their environment or their relationship to it. The paradigmatic example being the beaver, which significantly alters the local environment by building a dam, and consequently altering the selective environment it experiences. Both the Baldwin effect and niche construction are central elements in the discussion of an extended evolutionary synthesis. The argument for an extended synthesis from niche construction theory is that viewing organisms as merely passive objects that are filtered by natural selection neglects the active role of the organism in its evolution (Odling-Smee 2003). They see niche construction as an evolutionary process whereby the activities of organisms counter or direct the action of natural selection. Consequently, they argue that niche construction should be seen as a potentially equally important evolutionary process as natural selection itself. The same is often said of the Baldwin effect. It constitutes a corollary process of selection (viz. organic selection) and is often considered to be an evolutionary mechanism or process (Bateson 2017a, 2017b).

According to the adherents of an extended evolutionary synthesis, we need to pay more attention to the neglected process of niche construction, organic selection and other processes where organisms play an active role in evolution. Allowing more processes to be considered evolutionary processes is one way we can do this (Scott-Phillips et al. 2014; Laland 2015). However, this solution has been met with some skepticism (e.g. Welch 2017; Scott-Phillips et al. 2014), as it is unclear whether granting something the status of 
an evolutionary process actually increases our understanding of evolution. Another problem with viewing niche construction as an evolutionary process that counteracts natural selection is that it still treats natural selection as an asymmetrically externalist environmental process. If niche construction "counteracts" the action of selection, selection must be a process that runs from the environment to the organism. Instead, we should start from an ecological metaphysics of evolution (Walsh 2015).

\section{An Ecological Metaphysics of Evolution and Organism-Environment Interactions.}

When Walsh (2015) calls for an ecological metaphysics of evolution, he highlights that we might have missed a lot in our understanding of evolution by not seeing organisms as active (and purposive) agents in their environments. Treating organisms as biological agents prior to evolutionary agents is a necessary step in the direction of an ecological metaphysics (Walsh 2015). Biological entities are entities that interact with their environment. The relationship between the organism and the environment is crucial and in a sense prior to both the organism and environment themselves. Without any organisms there would be no environments, and conversely, without environments there would be no organisms (Lewontin 2000). From an ecological metaphysics of evolution, then, the fundamental unit is that of organism-environment interactions. Evolution concerns changes in the types of interactions there are. Mostly these interactions change in virtue of changes to the organism itself, for example by organism evolving faculties with which they interact with their environment in novel ways. Such kinds of changes to organism-environment interaction are captured by the theoretical framework offered by the modern synthesis. 
However, an environment can also change in such a way that organism-environment interactions change as a result, and more importantly, an organism can change the environment or its relationship to it such that the organism-environment interactions change (i.e., niche construction).

Natural selection, then, is the process whereby organism-environment interactions are preferentially selected. It is concerned with the outcomes of organism-environment interactions over the life-history of an organism (or at least to the end of its reproductive age) relative to those of its population. The strength of and response to selection is determined by the probability that advantageous interactions reoccur in subsequent generations. Consequently, advantageous hereditary traits (traits that are passed on through genetic inheritance) are more likely to spread than acquired traits whose likelihood of reoccurrence is lower. But it is in principle possible for selection to act on advantageous organism-environment interactions that are acquired (e.g., as a result of niche construction or behavioral plasticity).

Take, for instance, gastrolith usage. Gastroliths are small stones that are ingested and then reside in the gastrointestinal tract of some animals. Carrying gastroliths is certainly an example of an acquired trait, as it is something the animal has to acquire from its environment to utilize. Usage of gastroliths is quite common among some groups of vertebrates and may serve a wide variety of different functions in relation to different environments (Wings 2007). For example, some have argued that in aquatic environments gastroliths might be used as ballast or for buoyancy control (Rondeau et al. 2005). While in 
terrestrial environments some have argued that gastroliths may supply minerals and help with trituration and mixing of foodstuffs (Wings 2007).

If, for instance, an organism enjoys a higher fitness relative to other members of its population as a result of having ingested gastroliths, natural selection will favor that individual. Further, let us say that this organism learnt to ingest gastroliths by observing its parents and continue the habit of ingesting such stones. If in the subsequent generations gastrolith ingestion is reliably transmitted through observational learning, and the fitness advantage is sufficiently high, natural selection could spread this trait throughout the population. Natural selection could also favor those who have a disposition for ingesting gastroliths, with or without observational learning, making it an acquired trait with a hereditary basis (which is an example of the Baldwin effect). For natural selection, however, the basis on which the gastrolith is ingested — be it by way of learning or instinct - is irrelevant as long as the stone is ingested. It is the outcome of the interactione.g., the improved trituration of foodstuffs - which is conducive to the fitness advantage, not whether or not it is learnt or instinctual, as long as the stone is reliably ingested. ${ }^{3}$ More generally, we could say that the primary way in which genes matter for selection is in how conducive they are to the reliability and likelihood of advantageous organism-environment

\footnotetext{
${ }^{3}$ Of course, if all members of a population ingest gastroliths, and some do it instinctually while others need to learn it through observation, natural selection will most likely favor the instinctual response because the trait itself (i.e., gastrolith ingestion) is presumably transmitted with a higher fidelity if it is congenital rather than learned.
} 
interactions to reoccur in subsequent generations. Taking this perspective on how natural selection acts, let us return to how we should interpret niche construction and the Baldwin effect. Are they different selective processes, as it is commonly argued?

5. Niche Construction and the Baldwin Effect Revisited. Both niche construction and the Baldwin effect have been seen as distinct evolutionary mechanisms or processes (e.g., Odling-Smee et al. 2003; Bateson 2017a, 2017b). Some even go as far as saying that they are different selective processes, as when niche construction is interpreted as a process where organisms counteract natural selection by modifying selection pressures (Laland 2015). The Baldwin effect is seen as a distinct selective process which operates on acquired traits until there is genetic variation present so natural selection can "take over" and consequently turn them into congenital traits.

I think these interpretations are misguided, and stem from viewing natural selection as a process of environmental filtration concerned with primarily with genes, i.e., from a "molecular" metaphysics of evolution. If we instead take the point of view introduced above, where natural selection is concerned with the outcomes of organism-environment interactions and their relative reoccurrence, niche construction and the Baldwin effect are ways in which adaptation can occur and consequently be selected for. Niche construction is one way in which an organism can achieve a fitness advantage relative to other members of its population, but it is not a process that is counteracting the effects of natural selection. As long as the niche constructing behavior reoccurs reliably and the altered ecological conditions are reliably transmitted across generations it is no different from any other 
phenotypic trait in relation to natural selection. Acquired traits, and the Baldwin effect more generally, are also not selected initially by a process distinct from natural selection (i.e., organic selection). They are selected for by natural selection from their first occurrence, it is just a shift in the system of inheritance that is responsible for the reoccurrence of the trait. Sometimes, it makes sense to say that an acquired trait has become a congenital trait, as for instance when a learnt behavior has become instinctual. However, in the case discussed above, the ingestion of gastroliths, it is unclear if it can ever fully be a congenital trait, as the key feature of having that trait is to acquire a suitable rock from the environment (though the disposition can certainly be congenital).

Natural selection understood as a process acting on the outcome of reoccurring organism-environment interactions has the benefit of being compatible with the main insights of the modern synthesis, while also allowing for other cases to be included as ways in which organism-environment interactions can change and be acted on by selection, such as niche construction and the Baldwin effect. It explains why the genetic system of inheritance is so important - because it is a system which is necessary for the development of (most, if not all) phenotypes, and consequently for there to be any organismenvironment interactions at all. While simultaneously explaining how certain behavioral innovations, cultural traits, etc. can be selected for by natural selection, without being (directly) dependent on genetic variation or inheritance.

6. Conclusion. I have argued that natural selection is standardly understood as a process of environmental filtration concerned primarily with genes. Further, I followed Walsh (2015) 
in arguing that this stems from a gene-centered and externalist ("molecular") metaphysics of evolution. If we instead opt for an ecological metaphysics of evolution our understanding of natural selection becomes different. On such a metaphysics of evolution, natural selection becomes a process that acts on the outcomes of the advantageous interactions an organism has with its environment during its life-history. As long as such interactions reoccur reliably in subsequent generations, natural selection will be insensitive as to what brings about these interactions, be it through genetic inheritance, social learning, cultural transmission, etc. A benefit of this view is that the ecological account of natural selection is compatible with the main insights from the modern synthesis, while also allowing for phenomena traditionally excluded from the modern synthesis, but emphasized by the extended evolutionary synthesis. Finally, the ecological view of natural selection can integrate some of these novel phenomena easily, without having to supplement and extend evolutionary theory with a host of new evolutionary processes.

\section{REFERENCES}

Anholt, R. and Mackay, T. 2010. Principles of Behavioral Genetics. London: Elsevier Academic Press.

Avital, E. and Jablonka, E. 2000. Animal Traditions: Behavioural Inheritance in Evolution. Cambridge: Cambridge University Press.

Baldwin, J. M. 1896a. “A New Factor in Evolution.” American Naturalist. 30: 441-451. 
Baldwin, J. M. 1896b. “A New Factor in Evolution (Continued).” American Naturalist. 30: $536-553$.

Bateson, P., 2004. "The Active Role of Behaviour in Evolution." Biology and Philosophy. 19: $283-298$.

Bateson, P., 2017a. “Adaptability and Evolution.” Interface Focus 7.

Bateson, P., 2017b. Behaviour, Development and Evolution. Cambridge: Open Book Publishers.

Bateson, P. and Gluckman, P. 2011. Plasticity, Robustness, Development and Evolution. Cambridge: Cambridge University Press.

Brandon, R. N. 1990. Adaptation and Environment. Princeton, NJ: Princeton University Press.

Dawkins, R. 1976. The Selfish Gene. Oxford: Oxford University Press.

Dawkins, R. 1978. "Replicator Selection and the Extended Phenotype." Ethology. 47(1): $61-76$.

Dawkins, R. 1982. The Extended Phenotype. Oxford: Oxford University Press.

Dretske, F. 1988. Explaining Behavior: Reasons in a World of Causes. Cambridge, MA: The MIT Press.

Futuyma, D. J. and Kirkpatrick, M., 2017. Evolution. Sunderland, MA: Sinauer Associates Inc.

Gilbert, S. F. and Epel, D. 2015. Ecological Developmental Biological: The Environmental Regulation of Development, Health, and Evolution. Sunderland, MA: Sinauer Associates Inc. 
Godfrey-Smith, P. 1996. Complexity and the Function of Mind in Nature. Cambridge: Cambridge University Press.

Godfrey-Smith, P. 2009. Darwinian Populations and Natural Selection. Oxford: Oxford University Press.

Jablonka, E. and Lamb, M. J. 2014. Evolution in Four Dimension: Genetic, Epigenetic, Behavioral, and Symbolic Variation in the History of Life. Cambridge, MA: The MIT Press.

Krebs, J. R. and Davies, N. B. 1993. An Introduction to Behavioural Ecology. Malden, MA Blackwell Science Ltd.

Laland, K. N., 2015. “On Evolutionary Causes and Evolutionary Processes.” Behavioral Processes. 117: 97-104.

Laland, K. N., Feldman, M. W., Müller, G. B., Jablonka, E. Uller, T., Sterelny, K., Moczek, A., Odling-Smee, J., 2014. "Does Evolutionary Theory Need a Rethink? Yes, Urgently.” Nature. 514(7521): 161-164.

Lehrman, D. S. (1970). "Semantic and Conceptual Issues in the Nature-Nurture Problem." In L. R. Aronson, E. Tobach, D. S. Lehrman, and J. S. Rosenblatt (eds.), Development and Evolution of Behavior: Essays in Memory of T. C. Schneirla. , San Francisco: W. H. Freeman, pp. 17-52.

Levis, N. A. and Pfennig, D. W. 2016. "Evaluating 'Plasticity First Evolution in Nature: Key Criteria and Empirical Approaches.” Trends in Ecology and Evolution. 31(7): $563-574$. 
Lewontin, R. C. 1970. “The Units of Selection.” Annual Review of Ecology and Systematics. 1: 1-18.

Lewontin, R. C. 1983. "The Organisms as the Subject and Object of Evolution." Scientia. 118: $63-82$.

Lewontin, R. C. 2000. The Triple Helix: Gene, Organism, and Environment. Cambridge, MA: Harvard University Press.

Matthen, M. and Ariew, A. 2002. "Two Ways of Thinking about Fitness and Natural Selection. Journal of Philosophy. 99(2): 55-83.

Millstein, R. L. 2009. “Populations as Individuals.” Biological Theory. 4(3): 267-273.

Morgan, C. L. 1896. Habitat and Instinct. New York, NY: Edward Arnold Press.

Odling-Smee, J., Laland, K. N., and Feldman, M. W. 2003. Niche Construction: The Neglected Process in Evolutionary Theory. Princeton, NJ: Princeton University Press.

Okasha, S. 2006. Evolution and the Levels of Selection. Oxford: Oxford University Press.

Osborn, H. F. 1896. “A Mode of Evolution Requiring Neither Natural Selection nor the Inheritance of Acquired Characters." Transactions of the New York Academy of Sciences. 15: 141-142.

Otsuka, J. 2016. "Causal Foundations of Evolutionary Genetics.” The British Journal for the Philosophy of Science. 67(1): 247-269.

Oyama, S. 2000. The Ontogeny of Information: Developmental Systems and Evolution. Durham, NC: Duke University Press.

Piaget, J. 1978. Behavior and Evolution. New York: Pantheon Books. 
Pigliucci, M. and Müller, G. B. 2010. Evolution: The Extended Synthesis. Cambridge, MA: The MIT Press.

Ramsey, G. 2013a. “Can Fitness Differences Be a Cause of Evolution?” Philosophy and Theory in Biology. 5:e401.

Ramsey, G. 2013b. “Organisms, Traits, and Population Subdivisions: Two Arguments Against the Causal Conception of Fitness?" British Journal for the Philosophy of Science. 64: 589-608.

Ramsey, G. 2016. “The Causal Structure of Evolutionary Theory.” Australasian Journal of Philosophy. 94: 421-434.

Rondeau, S. L. and Gee, J. H. 2005. "Larval Anurans Adjust Buoyancy in Response to Substrate Ingestion.” Copeia. 2005(1): 188-195.

Rosenberg, A. 1983. "Fitness.” Journal of Philosophy. 80(8): 457-473.

Scott-Phillips, T. C., Laland, K. N., Shuker, D. M., Dickins, T. E., and West, S. A., 2014. “The Niche Construction Perspective: A Critical Appraisal.” Evolution. 68(5): $1231-1243$.

Simpson, G. C. 1953. “The Baldwin Effect.” Evolution. 7: 110-117.

Sober, E. 1984. The Nature of Selection: Evolutionary Theory in Philosophical Focus. Chicago, IL: The University of Chicago Press.

Sultan, S. E. 2015. Organism \& Environment: Ecological Development, Niche Construction, and Adaptation. Oxford: Oxford University Press.

Walsh, D. M. 2010. "Not a Sure Thing: Fitness Probability, and Causation.” Philosophy of Science. 77(2): 147-171. 
Walsh, D. M. 2015. Organisms, Agency, and Evolution. Cambridge: Cambridge University Press.

Welch, J. J. 2017. "What's Wrong with Evolutionary Biology?” Biology and Philosophy. 32(2): 263-279.

West-Eberhard, M. J. 2003. Developmental Plasticity and Evolution. Oxford: Oxford University Press.

Williams, G. C. 1966. Adaptation and Natural Selection: A Critique of Some Current Evolutionary Thought. Princeton, NJ: Princeton University Press.

Wings, O. 2007. “A Review of Gastrolith Function with Implications for Fossil Vertebrates and a Revised Classification.” Acta Palaeontologica Polonica. 52(1): $1-16$.

Wray, G. A., Hoekstra, H. E., Futuyma, D. J., Lenski, R. E., Mackay, T. F. C., Schluter, D. and Strassmann, J. E. 2014. "Does Evolutionary Theory Need a Rethink? No, All is Well." Nature. 514: 161-164. 